\title{
Participación social en salud: la experiencia del programa de salud materna Arranque Parejo en la Vida
}

\author{
Emanuel Orozco-Núñez, M Antrop Soc,(') Miguel Ángel González-Block, PhD Sociol,(I) \\ Luz María Kageyama-Escobar, PhD Sal Púb,(I) Bernardo Hernández-Prado, PhD Sal Púb.(2)
}

\author{
Orozco-Núñez E, González-Block MA, \\ Kageyama-Escobar LM, Hernández-Prado B. \\ Participación social en salud: \\ la experiencia del programa de salud materna \\ Arranque Parejo en la Vida.
} Salud Publica Mex 2009;5 I:I04-I I3.

\section{Resumen}

Objetivo. Evaluar la implementación de las medidas participativas y la conformación de redes de apoyo para mujeres embarazadas. Material y métodos. Se realizó una evaluación cualitativa y comparativa en cuatro estados. Resultados. La coordinación y la participación comunitaria fueron favorables en los casos en los que se observó mayor asignación y disponibilidad de recursos, tales como transporte y posadas maternas. La participación y el liderazgo de actores gubernamentales favorecieron la vinculación y coordinación. El apoyo más valorado por las usuarias provino de su red de parentesco. Conclusiones. Es fundamental fortalecer y promover medidas participativas en zonas con elevada mortalidad materna. La amplia valoración de las redes de parentesco, las parteras y el voluntariado en los albergues sugiere que estos actores son un componente funcional de la red de apoyo y que es insuficiente focalizar la red en los servicios de salud y las autoridades municipales.

Palabras clave: participación social en salud; políticas de salud; redes de servicios de salud; México

\section{Orozco-Núñez E, González-Block MA,}

Kageyama-Escobar LM, Hernández-Prado B.

Social participation in health.

The expericience of the mexican maternal health

care program Arranque Parejo en la Vida.

Salud Publica Mex 2009;5 I: 104-I I3.

\section{Abstract}

Objective.To evaluate the implementation of its participative strategies and the creation of support networks for poor pregnant women. Material and Methods.A qualitative and comparative evaluation was carried on in four states. Results. Coordination and community participation were relevant in relation with major resources allocation and availability, particularly housing and transportation. Governmental actors involvement and leadership favoured linking and coordination. Pregnant women used to valuate as the major support source the one provided by their kinship networks. Conclusions. To strengthen and to stimulate participative strategies is fundamental in zones with high maternal mortality rates. The wide appreciation of kinship networks, midwives and voluntaries' support to pregnant women in housing and transportation, suggests that these actors are a functional component of the support network; it is insufficient focusing the support network on health services and municipal authorities.

Key words: social participation in health; health policy; health services networks; Mexico

(I) Centro de Investigación en Sistemas de Salud, Instituto Nacional de Salud Pública, México.

(2) Centro de Investigación en Salud Poblacional, Instituto Nacional de Salud Pública, México.

Fecha de recibido: 6 de febrero de 2008 - Fecha de aprobado: 22 de enero de 2009 Solicitud de sobretiros: Mtro. Emanuel Orozco Núñez. Instituto Nacional de Salud Pública. Av. Universidad 655, col. Santa María Ahuacatitlán. 62508 Cuernavaca, Morelos, México. Correo electrónico: eorozco@insp.mx 
$\mathrm{L}^{\mathrm{a}}$ mortalidad materna se ha reconocido como un problema del subdesarrollo en el ámbito global, razón por la cual su disminución se convirtió en una prioridad para las Naciones Unidas y se incluyó como uno de los ocho Objetivos de Desarrollo del Milenio. ${ }^{1}$ Como país participante en esta iniciativa, el gobierno mexicano se propuso reducirla en 35\% durante el periodo de 2001 a 2006, mediante la institución del programa Arranque Parejo en la Vida (APV), creado en 2001 para atender desafíos de salud materna en estados de alta prioridad. ${ }^{2,3}$ La cobertura de sus acciones se extendió de manera paulatina a otras entidades federativas, hasta que el Consejo de Salubridad General acordó su obligatoriedad para instituciones públicas y privadas del país mediante un acuerdo emitido por él a finales de $2004 .{ }^{4}$ Asimismo, el Plan Nacional de Salud 2007-2012 retomó entre sus metas dar continuidad a la disminución de la mortalidad materna en municipios de alta prioridad y presenta como medida para tal fin el fortalecimiento de las políticas de salud materna y perinatal. ${ }^{5}$

El diagnóstico realizado por el sector salud al inicio de la administración 2001-2006 mostró que los mayores retos de salud materna y perinatal se encontraban en las regiones con mayor pobreza y marginación. ${ }^{2}$ Ante ello, el diseño general del APV propuso la instrumentación federal y estatal de dos grandes paquetes de acciones para su atención: los componentes sustantivos, con acciones dirigidas a la vigilancia epidemiológica activa de los indicadores de condiciones de salud materna (atención médica del embarazo, parto y puerperio) y los estratégicos, con acciones orientadas a favorecer la coordinación de una red para fortalecer la organización comunitaria y mejorar la referencia oportuna de mujeres embarazadas a los servicios de salud. ${ }^{3}$

La red del APV está integrada por los servicios de salud y los servicios de apoyo social: "... La red de servicios de salud incorpora la atención desde el primer nivel, incluyendo las parteras, brigadas, unidades de salud y unidades móviles". Por su parte, la red de apoyo social "... inicia sus acciones desde la comunidad con la promoción entre la población de estilos de vida saludables, (...) involucrando también a las autoridades municipales y grupos organizados de la comunidad, para la referencia a la Clínica (...) que corresponda...".3

En este escrito se presentan los resultados de la evaluación de APV que llevó a cabo el Instituto Nacional de Salud Pública en 2006, la cual tuvo un componente de procesos y otro de análisis de impacto. Este análisis corresponde al componente de procesos y se enfoca en la implementación de formas de participación comunitaria para la prevención de la mortalidad materna en cuatro estados de México. El artículo describe las características observadas de la red de servicios y revisa las determi- nantes locales de la participación comunitaria que le confieren diversos grados de funcionalidad.

Una red social puede entenderse como una forma natural de organización entre individuos, ${ }^{6}$ para desarrollar funciones específicas a partir de un motivo explícito y un efecto implícito en su estructura. ${ }^{7}$ El modelo conceptual del APV se analiza como una red social, lo que hace posible caracterizar su funcionamiento en los estados seleccionados en torno de la referencia de mujeres embarazadas a unidades de atención.

Las acciones del APV relacionadas con la participación comunitaria se concibieron para promover la vinculación de comunidades y autoridades locales con los servicios de salud, con objeto de instalar y operar transporte y posadas para la atención a las mujeres embarazadas. También se buscó garantizar la articulación de los servicios médicos institucionales de todos los niveles, así como los servicios comunitarios existentes mediante la coordinación del apoyo social ante situaciones de emergencia. ${ }^{3}$ La evaluación realizada se propuso identificar el cumplimiento de estos objetivos a la luz de la perspectiva de actores de los niveles estatal y local, las características de los contenidos de la participación social y los procesos y apoyos documentados.

Los resultados obtenidos pueden tener implicaciones en la focalización de acciones, la equidad, la provisión de servicios de salud y el tipo de medidas de suministro de servicios para grupos vulnerables. Se considera que las oportunidades y retos documentados pueden favorecer la conformación de redes de apoyo comunitarias efectivas para la aplicación de medidas que impliquen la participación comunitaria para el cumplimiento de sus objetivos.

\section{Material y métodos}

Se realizó un estudio cualitativo transversal y comparativo. La recolección de la información se realizó de octubre de 2005 a enero de 2006 en los estados de Chiapas, Guerrero, Hidalgo y Sonora, cuya selección se efectuó tras contrastar contextos de alta y baja mortalidad materna y desempeños eficientes y deficientes de los servicios estatales de salud. Este criterio permitió incluir a entidades con un buen desempeño de las iniciativas del APV y estados con una aplicación deficitaria. Chiapas y Guerrero tuvieron durante el periodo 2000-2004 las tasas de mortalidad materna e infantil más altas del país. Hidalgo fue uno de los pocos casos que instituyó la totalidad de las acciones del APV, mientras que Sonora no recibió apoyo federal directo del APV y notificó una de las tasas de mortalidad materna e infantil más bajas de México. ${ }^{8}$ 
La evaluación buscó contrastar experiencias y lecciones que permitieran la utilización de los resultados para el aprendizaje mediante la difusión de prácticas específicas; ${ }^{9}$ con ese fin se usó un criterio diferenciado para la selección de las unidades de observación. En todos los estados se trabajó en las jurisdicciones sanitarias de las capitales dado que concentraban las unidades de mayor capacidad y eran las cedes administrativas de los servicios. Este ejercicio se complementó con la selección de jurisdicciones rurales en las que había unidades utilizadas por población rural e indígena. Es importante señalar que en los casos de Guerrero y Chiapas, las jurisdicciones rurales seleccionadas respondieron a una situación intencional. En el primer caso se seleccionó la que notificó la tasa de mortalidad materna más alta del país, mientras que en Chiapas se seleccionó la que comunicó el mayor grado de cambio. No obstante sus contextos de pobreza, y pese a que en ambas hubo referencia de apoyos de distintos órdenes de gobierno, la jurisdicción seleccionada en Chiapas resultó relevante por ser además un enclave del Ejército Zapatista. En cada jurisdicción sanitaria se seleccionaron un hospital y dos centros de salud vinculados por el sistema de referencia y contrarreferencia local.

Se integró una brigada multidisciplinaria compuesta por dos médicas, dos antropólogas, una administradora de servicios de salud y un analista de políticas de salud. Este personal aplicó las siguientes técnicas de investigación: observación directa no participativa, entrevistas semiestructuradas, listas de verificación y mapeo político. Los universos de estudio y las actividades de campo se dividieron en dos grupos:

1. Servicios de salud. Se visitaron cuatro sedes de sistemas estatales de salud, ocho centros de salud, cuatro hospitales integrales rurales, cuatro hospitales estatales, tres posadas maternas y ocho oficinas jurisdiccionales.

2. Población. Se entrevistó, previo consentimiento informado, a 80 usuarias embarazadas y puérperas, 17 parteras, ocho funcionarios municipales, 20 proveedores de primer y segundo niveles de atención, nueve decisores jurisdiccionales, ocho decisores estatales y siete decisores federales. Con estos informantes se indagaron experiencias de participación comunitaria para el traslado de urgencias obstétricas mediante redes de apoyo.

El análisis de la información se realizó mediante matrices de relación de variables y codificación por medio del software Atlas-Ti. Las entrevistas se analizaron por tipo de informante y se emplearon como criterios de codificación palabras clave relacionadas con temas específicos de cada acción evaluada. Se establecieron gradientes de desempeño basados en los testimonios de los informantes, los cuales se complementaron con observaciones de campo e información secundaria de los servicios estatales de salud.

\section{Resultados}

Para analizar las dimensiones de la participación comunitaria en relación con el APV, se identificó a actores y procesos que favorecieran la disponibilidad de transporte y albergue para las mujeres embarazadas y sus hijos menores, como parte del proceso de atención de episodios obstétricos y pediátricos. Se exploró el papel de las autoridades estatales y municipales en el apoyo a la referencia de casos complicados, así como los acuerdos suscritos entre estas autoridades y los proveedores de servicios comunitarios. También se documentaron las experiencias de apoyo informadas por las usuarias y la participación de parteras tradicionales en zonas de difícil acceso en los cuidados maternos.

\section{Actores y procesos de la participación comunitaria}

Los principales actores identificados en los cuatro estados en cuanto al apoyo comunitario de problemas obstétricos fueron: tomadores de decisiones estatales y jurisdiccionales, proveedores de servicios de primer nivel, proveedores de servicios de segundo nivel, autoridades municipales, transportistas, voluntarios, parteras, parientes y usuarias. La vinculación de los actores fue más favorable donde había mayor asignación y disponibilidad de recursos (cuadro I).

Los estados en los que se observaron efectos más favorables de la vinculación de actores fueron Chiapas y Sonora, donde autoridades, parientes, voluntarios y usuarios apoyaron la referencia de urgencias obstétricas, la identificación de mujeres embarazadas en zonas aisladas y la referencia de éstas a los servicios de salud. Se verificó que en ambos casos estos apoyos los identificara la población que habitaba las áreas de más difícil acceso.

Los principales apoyos gubernamentales en tres estados fueron la asignación de ambulancias a los ayuntamientos (se encontraron en todos los casos testimonios de munícipes que favorecieron el apoyo de las acciones del APV para la vigilancia del embarazo), la referencia oportuna de casos complicados y el apoyo a parteras tradicionales. Personal de las unidades de salud de esos estados señaló también que se coordinó 


\begin{tabular}{|c|c|c|c|c|}
\hline & & Cuadro I & & \\
\hline & ReCursos & DERIVADOS DE LA VINCULACIÓN DE & ACTORES & \\
\hline Estado & Recursos gubernamentales & Voluntariado & Apoyos municipales & Proceso favorecido \\
\hline Chiapas & $\begin{array}{l}\text { - Nómina municipal } \\
\text { - Apoyos extraordinarios de programas } \\
\text { estatales } \\
\text { - Ambulancias municipales }\end{array}$ & $\begin{array}{l}\text { - Aseo de las posadas } \\
\text { - Acompañamiento a las embarazadas } \\
\text { - Apoyos materiales de vecinos }\end{array}$ & - Pago de choferes & $\begin{array}{l}\text { - Referencia de casos } \\
\text { - Censos de } \\
\text { embarazadas }\end{array}$ \\
\hline Guerrero & - Fondos municipales & - Comités de transporte & - Pago de choferes & - Referencia de casos \\
\hline Hidalgo & - Ambulancias municipales & $\begin{array}{l}\text { - Apoyo de la Secretaría de } \\
\text { Seguridad Pública }\end{array}$ & - Pago de choferes & - Referencia de casos \\
\hline Sonora & $\begin{array}{l}\text { - Vehículos del ayuntamiento } \\
\text { - Ambulancias municipales } \\
\text { - Apoyos extraordinarios de programas } \\
\text { estatales }\end{array}$ & $\begin{array}{l}\text { - Comités de transporte } \\
\text { - Aseo de las posadas }\end{array}$ & $\begin{array}{l}\text { - Transporte de brigadas } \\
\text { de salud } \\
\text { - Pago de choferes } \\
\text { - Ambulancia aérea }\end{array}$ & $\begin{array}{l}\text { - Control prenatal } \\
\text { - Referencia de casos }\end{array}$ \\
\hline
\end{tabular}

con representantes de las comunidades mediante la formación de comités para el transporte y la referencia de pacientes.

Un aspecto relevante de la participación de actores del nivel estatal fue la gestión y asignación de recursos adicionales a los suministrados por la federación. En Chiapas y Sonora se notificó la vinculación de acciones del APV con otros programas estatales y federales, como Oportunidades y el Seguro Popular de Salud. En Chiapas se aplicó el programa estatal Vida Mejor y en Sonora la iniciativa Bienvenidos Todos los Nuevos Sonorenses. El primer caso consistió en un paquete de servicios de atención primaria a la salud financiado con recursos estatales para municipios con muy alta marginación. El segundo se refirió a una serie de servicios de atención del embarazo, el parto y la salud de los recién nacidos auspiciado también por el gobierno estatal. En realidad, el APV no operó como tal en Sonora ni contó con recursos federales. Más aún, el gobierno de Sonora donó a los servicios estatales de salud una ambulancia aérea, en virtud de las enormes distancias por cubrir en el estado. En ambos casos, actores comunitarios y usuarias comentaron que se han desarrollado mecanismos para una identificación más eficiente de las mujeres embarazadas con riesgos de complicaciones, como la vigilancia por parte de vecinas y parteras comunitarias. Estos programas resultaron relevantes porque tuvieron componentes de asistencia a pacientes embarazadas e integraron padrones de usuarias, los cuales se valoraron en estos estados como confiables por parte de los proveedores de servicios y los trabajadores de los ayuntamientos.
En Guerrero hubo frecuentes comentarios sobre limitaciones en cuanto a la vinculación gubernamental y la ausencia de bases comunitarias de participación, como las descritas para los otros estados, si bien se notificaron casos de pago de choferes para el transporte de casos críticos.

El voluntariado informó mecanismos de participación y coordinación, como la creación de comités comunitarios para apoyar acciones de los servicios de salud. En Sonora, los comités funcionaron bajo la supervisión de los ayuntamientos, mientras que en Chiapas se coordinaron con los servicios de salud. Estos comités se conformaron con representantes de los servicios de salud y los ayuntamientos y se delinearon mecanismos para la disponibilidad de transporte y choferes. En Guerrero e Hidalgo se comunicó la creación de un fondo para pagos y asegurar con ello la solvencia de recursos para situaciones de emergencia.

En los cuatro estados, las autoridades municipales entrevistadas señalaron que habían apoyado los trabajos de los equipos de salud ambulatorios en comunidades de difícil acceso. Hidalgo fue la única entidad que recibió apoyo de la Secretaría de Seguridad Pública del gobierno estatal para la salvaguarda de la ambulancia.

Los retos de vinculación identificados se relacionaron con problemas de seguimiento sistemático de los casos referidos y barreras culturales de accesibilidad a servicios de salud en zonas de diversidad cultural. En el primer caso se encontró que no se usaban formatos ni mecanismos de control de las referencias de urgencias obstétricas en los ayuntamientos visitados. Por su parte, las mayores barreras para la vinculación con usuarias y 
otros componentes de los recursos comunitarios como las parteras se reconocieron en las jurisdicciones sanitarias donde se atendía a población rural e indígena.

\section{Instalación y operación de transporte y posadas maternas}

En este apartado se describen mecanismos y factores que favorecieron una coordinación eficiente del transporte de personas con urgencias obstétricas y la disponibilidad de posadas maternas. El APV establece que ambos recursos deben tener el apoyo de las comunidades, como resultado de la vinculación establecida entre los actores para un mejor acompañamiento de las usuarias en estado crítico por parte de familiares o parteras.

La estructura observada del servicio de transporte sugiere que hubo adaptación a las condiciones de cada estado y que desempeñó un papel importante la instalación adicional de sistemas de radiotelefonía para la atención de urgencias, como se notificó en Hidalgo y Sonora. En términos de su adecuación, en estos estados se observó que las ambulancias municipales estaban mejor equipadas. Chiapas fue el único estado en el que se encontró un sistema municipal de ambulancias, en el cual el gobierno estatal asignó una ambulancia equipada a todas sus cabeceras municipales.

No obstante la mención de su disponibilidad, en los cuatro estados se indicó que la demanda de traslados de mujeres embarazadas no era muy alta, por lo que este servicio se concedió a otras urgencias. Los informantes de los ayuntamientos de más difícil acceso abundaron en la importancia de este recurso, dadas las distancias y el tiempo que tomaba recorrerlas en condiciones adversas. Las situaciones más dramáticas proceden de Guerrero y Chiapas, donde se refirió que era necesario transportar a pie a las mujeres entre varias personas en hamacas o ca- millas de varas, debido a que en diversas circunstancias no fue posible el acceso a vehículos automotores.

En las entrevistas con usuarias se detectó el pago de cuotas por servicios de traslado en Sonora, Hidalgo y Guerrero. Chiapas fue el único caso en el que no se registraron pagos por servicio, puesto que se incluyó como parte de él el retorno de los beneficiarios a sus comunidades. Los montos de las erogaciones fluctuaron entre 20 y 200 pesos por viaje y fueron objeto de queja de algunas de las informantes.

En el cuadro II se presenta la situación encontrada en posadas seleccionadas al momento de la evaluación. Su disponibilidad se verificó en Sonora, Guerrero y Chiapas, tras observar que estuvieran en funcionamiento. Sólo en Guerrero se encontró la llamada "Posada AME" utilizada por el APV. En los casos de Chiapas y Sonora se emplearon denominaciones locales, como "Casa Materna" y "Posada Materna", respectivamente. Las posadas en los tres casos observados se localizaron en zonas contiguas a un hospital.

Se observó en Chiapas y Guerrero que las posadas estaban disponibles en las jurisdicciones sanitarias rurales seleccionadas, mientras que en Sonora la posada se encontró en la capital estatal. En los tres estados, las posadas funcionaban y daban servicios gratuitos a las usuarias y sus parientes. Las usuarias valoraron en buenos términos su disponibilidad .

Para su funcionamiento, el cuadro II muestra que las posadas se relacionaron con distintas instancias gubernamentales. En Sonora, la Posada Materna se halla frente al Hospital Infantil y el Hospital de la Mujer (ambos vecinos) y ofrece alojamiento a las mujeres, los niños y sus parientes; su operación tiene el apoyo del gobierno estatal. En Guerrero, la posada se encuentra en las instalaciones de la Comisión Nacional para el Desarrollo de los Pueblos Indígenas (antes Instituto

\begin{tabular}{|c|c|c|c|c|c|}
\hline \multicolumn{6}{|c|}{ Cuadro II } \\
\hline \multicolumn{6}{|c|}{ CARACTERÍSTICAS OPERATIVAS DE LAS POSADAS MATERNAS } \\
\hline Estado & Ubicación & Vinculación con servicios de salud & $\begin{array}{l}\text { Vinculación con otras } \\
\text { dependencias }\end{array}$ & $\begin{array}{l}\text { Disponibilidad } \\
\text { durante la evaluación }\end{array}$ & Denominación \\
\hline Chiapas & $\begin{array}{l}\text { - Comitán } \\
\text { - Las Margaritas }\end{array}$ & $\begin{array}{l}\text { - Hospital de Comitán } \\
\text { - Hospital Integral de Las Margaritas }\end{array}$ & Ayuntamientos & +++ & Casa Materna \\
\hline Guerrero & Tlapa & $\begin{array}{l}\text { - Hospital General de Tlapa } \\
\text { - Hospital de la Mujer }\end{array}$ & CONADEPI & ++ & Posada AME \\
\hline Sonora & Hermosillo & $\begin{array}{l}\text { - Hospital Infantil } \\
\text { - Hospital de la Mujer }\end{array}$ & Gobierno del estado de Sonora & +++ & Posada Materna \\
\hline
\end{tabular}


Nacional Indigenista) de Tlapa, frente al recién inaugurado Hospital de la Mujer. Se comentó que existían limitaciones financieras en su operación. En Comitán y Las Margaritas se identificaron las facilidades más completas para apoyar a las mujeres embarazadas y sus parientes y se concedió un apoyo notable por parte de los ayuntamientos. El logro de estos apoyos fue reconocido con el Premio de Excelencia APV en 2005 y 2006.

Las posadas maternas reconocidas al momento de la evaluación dieron servicio a un promedio de tres mujeres cada una; se observó además que las usuarias iban acompañadas en promedio por dos personas. Las usuarias entrevistadas indicaron que procedían de municipios cercanos a la ubicación de la posada y fue evidente asimismo la participación del voluntariado, parteras y familiares en su aseo y la preparación de los alimentos, los cuales estuvieron disponibles en los tres casos. En todas las oportunidades se informó que la atención no generaba pagos por parte de las usuarias.

\section{Articulación de servicios médicos $y$ apoyos comunitarios}

Explorar el funcionamiento de los servicios comunitarios fue uno de los aspectos más complejos de la evaluación. Se investigaron los convenios y los acuerdos locales que, en opinión de los informantes, dieron mayor sustento a la vinculación entre autoridades gubernamentales. También se indagó acerca del liderazgo de estos actores y la formalización de la colaboración y la disponibilidad de los recursos. La situación documentada se describe en el cuadro III.

Seis de los ocho informantes municipales entrevistados señalaron que fueron convocados por autoridades sanitarias para apoyar acciones de referencia y atención oportuna de mujeres embarazadas y urgencias obstétricas, en particular el transporte y las posadas maternas. Los altos desempeños notificados para Chiapas, Hidalgo

\section{Cuadro III \\ VINCULACIÓN DE AUTORIDADES MUNICIPALES CON APV

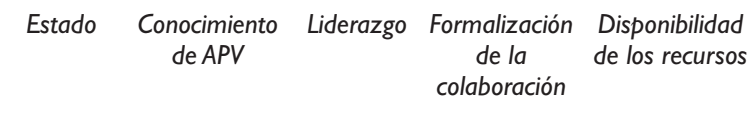

\begin{tabular}{lcccc} 
Chiapas & +++ & +++ & +++ & ++ \\
\hline Guerrero & ++ & + & ++ & + \\
\hline Hidalgo & +++ & ++ & + & ++ \\
\hline Sonora & ++ & +++ & ++ & +++
\end{tabular}

Desempeño: $+++=$ alto; ++ = medio; + = bajo y Sonora se atribuyeron a la participación de autoridades de distintos órdenes de gobierno, mientras que en Guerrero los mecanismos de coordinación mostraron un menor desarrollo.

Una de las dimensiones más reconocidas por proveedores y usuarias de servicios de salud fue el liderazgo de las autoridades en la conducción de las medidas de participación del APV. Ejemplo de ello en los servicios de salud fue la inclusión de los secretarios de salud en Chiapas y Sonora, quienes realizaron seguimiento regular de los casos críticos atendidos en ese momento. En Hidalgo, este reconocimiento fue más notorio en las autoridades municipales, debido a que financiaron al médico y las ambulancias.

En el ámbito municipal, los proveedores y usuarias reconocieron la colaboración de las autoridades en Chiapas, Hidalgo y Sonora. Una de las experiencias más desarrolladas de participación se observó en Chiapas, donde se institucionalizó la colaboración de los presidentes municipales en iniciativas participativas de salud materna como parte de los programas y políticas de bienestar social, a partir de la formulación de planes municipales de desarrollo. En estos planes, los presidentes municipales formalizaron el apoyo a la red municipal de ambulancias, la referencia de urgencias obstétricas a las clínicas de referencia, así como el mantenimiento de las unidades y el apoyo a las posadas y las capacitaciones para la acreditación de parteras.

En opinión de los proveedores de servicios de primer y segundo niveles, la participación y liderazgo de las autoridades favorecieron de manera notoria la vinculación y coordinación de actores clave. Al respecto, los munícipes de los estados evaluados se coordinaron de forma directa con los encargados de las unidades de atención para otorgar los apoyos para transporte y posadas maternas y agilizar la solicitud y la referencia de los pacientes.

Uno de los datos más relevantes sobre la coordinación de actores fue la referencia en Chiapas y Guerrero de la firma de convenios entre autoridades gubernamentales, sanitarias y municipales para formalizar el otorgamiento de los apoyos referidos a las usuarias y los servicios de salud.

Los mayores retos de continuidad se encuentran en el ámbito político. Al respecto, se mencionó que uno de los principales factores que influyeron en la interrupción del apoyo al transporte se vinculaba con cambios de autoridades municipales y estatales. Esto se corroboró en el terreno, debido a que el trabajo de campo coincidió con dos procesos electorales en entidades en las que se realizó esta evaluación; esto obligó a suspender temporalmente el otorgamiento de algunos de estos servicios. 
El saldo más negativo de estos procesos se notificó en una de las entidades, que no se menciona por razones de confidencialidad, en la cual proveedores de servicios y munícipes señalaron que el cambio de partido en el gobierno estatal modificó las jurisdicciones sanitarias y ello afectó la continuidad de los apoyos municipales. Esta alteración consistió en el cambio de áreas de adscripción del personal clave que ya se había capacitado en los componentes y las disposiciones del APV para la designación de nuevo personal. En contraste, un proceso similar en Chiapas tuvo un efecto más favorable: se observó que la presión ejercida por el propio gobierno estatal hacia el cumplimiento de objetivos de los programas municipales dio mayores resultados y se mantuvo la continuidad en el otorgamiento de apoyos.

Dado que el programa APV enfatiza que sus acciones están dirigidas en primera instancia a la atención de mujeres embarazadas que viven en condiciones de marginación y a sus hijos menores de dos años, se exploró con usuarias de estas características el apoyo recibido en su comunidad y en los servicios de salud durante el embarazo. Como parte de este ejercicio, se indagó en zonas rurales la vinculación de parteras tradicionales.

Resultó relevante que cerca de la tercera parte de las usuarias entrevistadas señalara barreras de accesibilidad geográficas, económicas y culturales a servicios de salud en los cuatros estados, situación que fue más recurrente en las jurisdicciones sanitarias con demanda significativa de población rural. No obstante que la mayor parte de las informantes recibió atención oportuna con una valoración favorable del trato recibido en las unidades, se recopilaron testimonios de usuarias en tres entidades que se sintieron maltratadas por parte de transportistas y proveedores tanto en el traslado a la unidad como en ésta.

La fuente de apoyo que tuvo la mayor valoración por parte de las usuarias en los cuatro estados provino de sus redes de parentesco. Este apoyo fue reconocido durante el embarazo, en la atención del parto y el cuidado de los niños menores. Si bien se refirió en la mayoría de los casos que el apoyo provenía de mujeres con distinto parentesco (madre, suegra, tía), se observó en las posadas maternas la presencia de acompañantes masculinos, casi siempre las parejas de las usuarias.

En cuanto a las parteras tradicionales, el APV establece la necesidad de su acompañamiento a las mujeres embarazadas de sus localidades, sobre todo en zonas donde la disponibilidad de personal médico es baja y existe demanda de estos servicios. Para su vinculación con las acciones del APV se estableció que las parteras debían ser capacitadas y acreditadas, para que participaran en la vigilancia de embarazos y el acompañamiento de las usuarias durante el parto y el puerperio.
Se encontró que en los cuatro estados había parteras tradicionales en funciones y que casi todas las parteras entrevistadas habían tenido capacitación en los servicios estatales de salud. Las parteras capacitadas recibieron un maletín con equipo básico (navajas, sábana, báscula, delantal, "sacaflemas", gasas). Para algunas de las entrevistadas este maletín fue una especie de incentivo, mientras que otras señalaron que su contenido no era suficiente para realizar su trabajo.

El apoyo otorgado por estas proveedoras pudo observarse en las posadas maternas; la experiencia más desarrollada y con mayor reconocimiento correspondió al estado de Chiapas. En este caso, las parteras entrevistadas señalaron una vinculación notable con los servicios de salud, favorecida por el apoyo de los presidentes municipales. Estos últimos fueron ampliamente identificados como patrocinadores de los programas de acreditación de parteras, por financiar el traslado desde su comunidad, su albergue, alimentación y los diplomas que les eran otorgados.

\section{Discusión}

La evaluación de los componentes participativos del APV permitió analizar las implicaciones relevantes acerca de la implementación de políticas de salud que requieren la vinculación y participación de actores clave, en este caso para la consecución de los objetivos de atención a la salud materna. Los resultados presentados muestran cierta heterogeneidad en las dinámicas locales que favorecieron la relación y coordinación de actores en los componentes participativos del programa. No obstante, se informó que en la mayor parte de los casos se aplicaron acciones participativas del APV con efectos favorables. Dado que no se dispuso de evidencia sobre el funcionamiento de estas redes en todo el país, la evaluación de jurisdicciones seleccionadas permitió extraer lecciones sobre condiciones relacionadas con su funcionamiento.

La información descrita evidencia dinámicas propias de una red social que respondieron a la demanda establecida por el programa; el objetivo de las acciones observadas es el otorgamiento de apoyo para la referencia de casos complicados, como lo expresaron diversos informantes (cuadro IV).

El análisis de los componentes participativos del $\mathrm{APV}$ en los estados muestra la relevancia de dos ámbitos de vinculación entre los actores participantes: el de los servicios y el de las comunidades. En el caso de los servicios, fue mayor la referencia a su participación donde hubo mayor relación entre proveedores de servicios de salud y munícipes, además de mayor conocimiento de los usuarios acerca de la forma de acceder al apoyo. Esta 
Cuadro IV

Desempeño de ACtORES Y PROCESOS POR ESTAdO

\begin{tabular}{|c|c|c|c|c|c|}
\hline \multirow[t]{2}{*}{ Actores } & \multirow[t]{2}{*}{ Procesos } & \multicolumn{4}{|c|}{ Desempeños estatales } \\
\hline & & Chiapas & Guerrero & Hidalgo & Sonora \\
\hline Funcionarios estatales & Apoyo gubernamental & +++ & + & ++ & +++ \\
\hline Munícipes & Apoyo a transporte y posadas & +++ & + & ++ & +++ \\
\hline Proveedores & Referencia y atención oportuna & +++ & + & + & +++ \\
\hline Voluntariado & Trabajo comunitario y asistencia en las posadas & +++ & + & NA & ++ \\
\hline Red de parentesco & Acompañamiento y apoyo en situaciones críticas & ++ & ++ & ++ & ++ \\
\hline Parteras & Acompañamiento y atención prenatal y del parto & +++ & + & + & + \\
\hline
\end{tabular}

Desempeño: $+++=$ alto; $++=$ medio; $+=$ bajo; $\mathrm{NA}=$ no aplica

coordinación entre proveedores y representantes municipales fue más amplia cuando se formalizó mediante convenios para su operación.

El hecho que, en promedio, la disponibilidad de transporte fuera más amplia en cabeceras municipales y unidades de segundo nivel, en comparación con el transporte con recursos humanos y tecnología rudimentarios que tuvo mayor referencia en comunidades rurales, sugiere la necesidad de acercar estos medios a dichas comunidades. El desarrollo de instalaciones comunitarias en zonas rurales requiere todavía un mayor esfuerzo, puesto que resulta notorio que en los casos en los que se concedió mayor respaldo a estas iniciativas se logró una mayor oferta de estos servicios.

El cuadro IV muestra desempeños contrastantes entre entidades con necesidades de salud materna similares, tal y como ocurre en Chiapas y Guerrero. Ambas entidades recibieron apoyo federal por considerarlas de alta prioridad, pero las acciones emprendidas en el nivel local favorecieron una mejora considerable de la atención en Chiapas. Una comparación de este comportamiento con la situación referida en Guerrero sugiere la necesidad de un mayor acompañamiento y fortalecimiento de capacidades locales para la atención de retos en materia de salud materna. Si bien las respuestas descritas sugieren la importancia del respaldo y la formalización de acuerdos, se encontraron barreras de continuidad para las nuevas administraciones municipales ante procesos electorales y cambio de autoridades de distintos niveles.

Respecto del ámbito comunitario, las usuarias expresaron como su principal fuente de apoyo el proveniente de sus redes de parentesco, el cual resultó complementario de las facilidades estudiadas y documentadas con munícipes y proveedores como parte del proceso de atención.

Un análisis más profundo de la vinculación entre los distintos actores de la red para lograr la referencia oportuna de las urgencias obstétricas indica que los alcances encontrados deben tomarse con cautela, dada la reciente aplicación del modelo de red social del APV y la fragilidad de los acuerdos con las autoridades municipales ante procesos políticos regionales. Sin duda, los recursos aportados por estos actores son importantes, pero su temporalidad respecto de otras formas de apoyo comunitario reconocidas por usuarias es más corta.

La vinculación entre los actores de la red social promovida por el programa APV se representa en la figura 1, en la cual se observa que el apoyo más relevante para las usuarias provino de sus redes de parentesco y que ésta es la relación con mayor contenido simbólico y cultural en la red. Este planteamiento concuerda con otros estudios que muestran que las personas mejor identificadas como proveedoras de apoyo están adscritas a la familia cercana, ${ }^{10}$ lo que sugiere que el programa podría aprovechar más este apoyo y los recursos que representa el cumplimiento de sus objetivos. El siguiente grado de vinculación local de las usuarias se establece con las parteras, en particular en zonas con población indígena y rural, y complementa al recibido por el personal de los servicios de salud. Los apoyos otorgados por los munícipes resultaron significativos por la movilización de recursos que éstos lograron. Sin embargo, se requieren mecanismos dirigidos para dar mayor permanencia a estos apoyos y sustento a la red APV. 

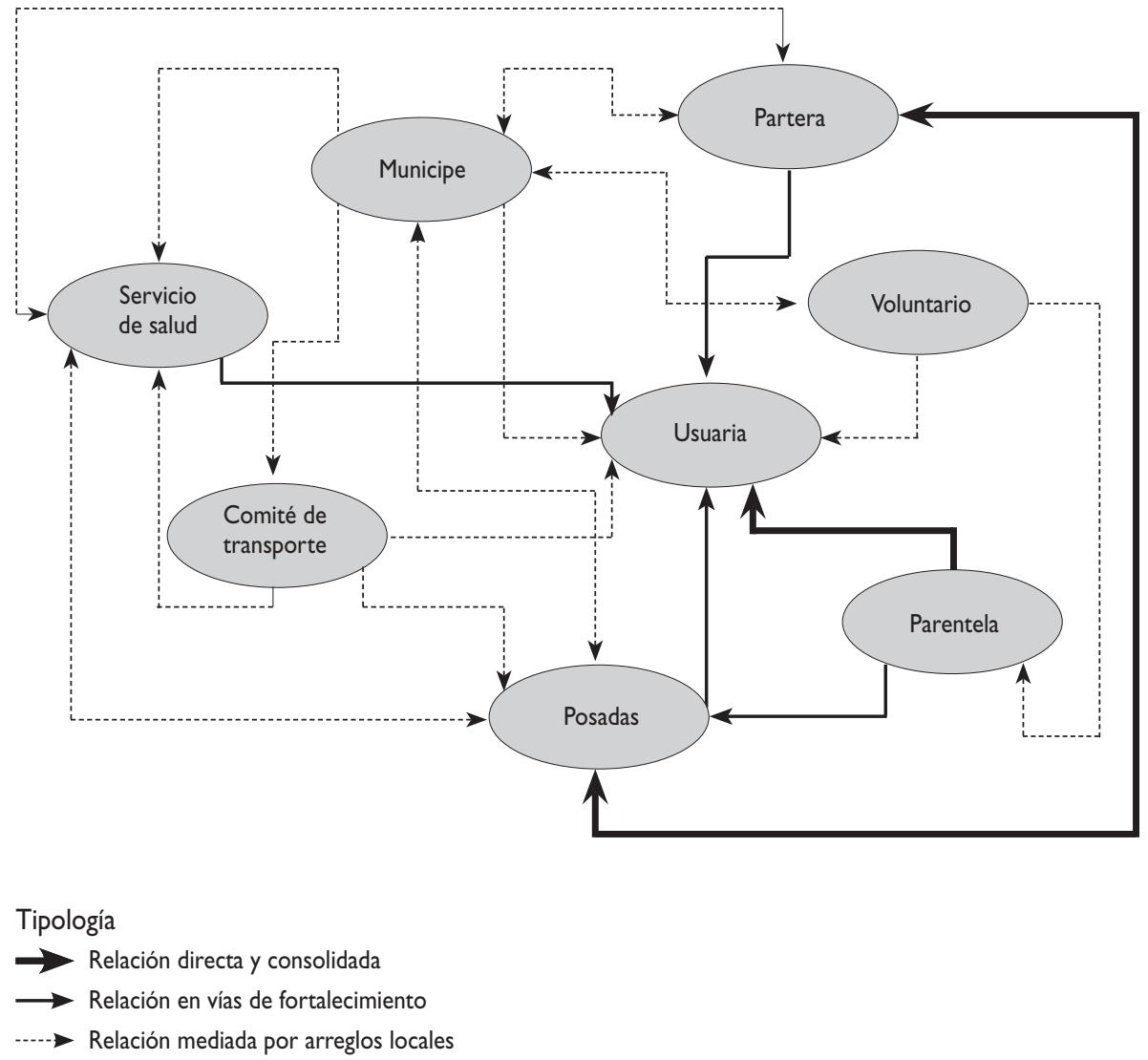

Figura I. La Red funcional

\section{Conclusiones}

La evaluación realizada a las acciones participativas del programa APV en las jurisdicciones sanitarias estudiadas sugiere que sus medidas para el apoyo de emergencias obstétricas influyeron en la disponibilidad de transporte y el albergue de mujeres embarazadas en posadas maternas. Este resultado fue notificado también en la certificación internacional de las acciones del APV, la cual destaca efectos potenciales del cumplimiento de estas disposiciones en la salud materna mediante la cobertura de la atención obstétrica y la mejora de la calidad de los servicios en esta materia. ${ }^{11}$

Hay coincidencia con otras fuentes ${ }^{12,13}$ en relación con la necesidad de fortalecer e incentivar estas medidas en las zonas con mayor mortalidad materna y en localidades rurales e indígenas. La amplia valoración de las redes de parentesco y de las parteras, así como del voluntariado en los albergues, señala la relevancia de haber incorporado estos recursos comunitarios como parte de la red social de apoyo para lograr un acompañamiento más efectivo y la detección oportuna de riesgos obstétricos. Resultaría insuficiente atribuir la funcionalidad del modelo a los servicios de salud y a las autoridades de distintos órdenes de gobierno.

Estas conclusiones pueden considerarse en el diseño de nuevos programas, tales como las "Caravanas de Salud" que aplicará la Secretaría de Salud dentro del gobierno en el periodo de 2006 a 2012. Con una mayor participación de los actores comunitarios se logrará una mayor equidad y se creará un sistema de salud más democrático.

\section{Agradecimientos}

Los autores agradecen la gran disponibilidad de las usuarias, el personal de los servicios de salud, los munícipes y las autoridades estatales para la realización de la investigación de campo. Reconocen también las facilidades otorgadas por el Centro Nacional de Equidad de Género y Salud Reproductiva para la evaluación de APV. 


\section{Referencias}

I. Gobierno de la República. Reporte de Objetivos de Desarrollo del Milenio. México: SEGOB, 2006.

2. Secretaría de Salud. Programa Nacional de Salud 200I-2006. La democratización de los servicios de salud en México. México: SSA, 2001. 3. Secretaría de Salud. Programa de acción Arranque Parejo en la Vida, Centro Nacional de Equidad de Género y Salud Reproductiva. México: SSA, 2003.

4. Consejo de Salubridad General. Decreto que establece la obligatoriedad de las acciones del programa Arranque Parejo en la Vida en instituciones de salud públicas y privadas. Diario Oficial de la Federación, I de noviembre de 2004.

5. Secretaría de Salud. Programa Nacional de Salud 2007-20 I2. Por un México sano: construyendo alianzas para una mejor salud. México: SSA, 2007. 6. Mendizabal E. Understanding networks. The functions of research policy networks. UK: Overseas Development Institute, 2005.

7. Mendizabal E. Building effective research policy networks: linking function and form. Working Paper 276. UK: Overseas Development Institute, 2006.
8. Secretaría de Salud. Salud México 200 I-2005. Información para la rendición de cuentas, Dirección General de Evaluación del Desempeño. México: SSA, 2006

9. González Block MA, Orozco E, Kageyama LM, Hernández B, et al. Evaluación del Programa de Acción Arranque Parejo en la Vida 200I-2005. Resumen Ejecutivo. México: Centro de Investigación en Sistemas de Salud, Instituto Nacional de Salud Pública, 2006.

10. Meléndez JC, Tomás JM, Navarro E.Análisis de las redes sociales en la vejez a través de la encuesta Manheim. Salud Publica Mex 2007;49: 408-4I4.

II. Secretaría de Salud, Fondo de Naciones Unidas para la Población, Organización Panamericana de la Salud. Certificación internacional del programa Arranque Parejo en la Vida. Informe Ejecutivo. México: SSAUNFPA, 2006.

12. Freyermuth G. Maternidad peligrosa. México: Centro Investigación de Estudios superiores en Antropología Social, 2004.

I3. FUNDAR. Mortalidad materna en México. México: Fundar AC, 2004. 